\section{Sisyphos beim Hürdenlauf?}

\author{
Inputorientierte Umweltpolitik zielt auf die Reduktion von Ressourcen und verspricht \\ die Lösung einer Reihe grundsätzlicher Umweltprobleme. Aus wirtschaftpolitischer \\ Perspektive steht die Umsetzung von inputorientierter Umweltpolitik allerdings vor \\ einer Reihe von Hindernissen wie Verteilungswirkungen, ordnungspolitischen Pro- \\ blemen und Schwierigkeiten der politischen Umsetzung die mit einer geeigneten \\ wirtschaftspolitischen Strategie aber überwunden werden können.
}

$\mathrm{D}$

Von Marcus Stewen dern ist erheblich: 4,3 Milliarden Tonnen schwer ist der Stoffstrom an Material, der im Jahr 2000 allein in Deutschland aus der Umwelt entnommen wurde. Das sind circa 52 Tonnen pro Kopf und etwa doppelt so viel Umweltressourcen wie 1960. Hinzu kommen circa 45,5 Milliarden Tonnen Wasser, eine Zunahme der Siedlungs- und Verkehrsfläche um circa 120 Hektar pro Tag (jährlich eine mehr als 40 Prozent größere Fläche als die Münchens) sowie erhebliche Mengen im Ausland induzierter Stoffströme. In der EU summierte sich der gesamte Materialverbrauch 1998 auf 18,7 Milliarden Tonnen ohne Fläche, Wasser und Luft. Insgesamt liegt der Verbrauch bei etwa 50 Tonnen pro Kopf (1). Dagegen wächst der materielle Ressourcenverbrauch der ärmeren Länder der Erde noch sehr langsam. Sollten ärmere Länder einmal das Verbrauchsniveau der Industrienationen erreichen, hätte das enorme Folgen für den globalen Ressourcenverbrauch und die Ökosphäre. Ein Stopp des absoluten Wachstums des Ressourcenverbrauchs ist jedoch nicht in Sicht, auch wenn in manchen Bereichen zeitweise eine relative Entkopplung einiger Stoffverbräuche vom Wirtschaftswachstum registriert werden konnte, die aber nicht zwangsläufig dauerhaft ist.

\section{Ressourceneinsparung durch} inputorientierte Umweltpolitik

Unterstuitzt wird der steigende Ressourcenverbrauch auch dadurch, dass sich Umweltpolitik seit ihrem Bestehen primär auf die Reduktion von Schad- und Gefahrstoffen und damit auf Emissionen, Abfälle und punktuelle Umweltprobleme konzentriert. Anders ausgedrückt: Umweltpolitik konzentriert sich auf die Outputseite der Ökonomie. Hinzu treten seit geraumer Zeit ökologische Schutzziele wie etwa der Schutz der Weltmeere oder der Atmosphäre.
Erste Ansätze eines politischen Paradigmenwechsel zeigten sich durch Einführung des Kreislaufwirtschaftsgesetzes und der verstärkten Forcierung von Energiesparmaßnahmen. Keiner dieser Ansätze aber konnte das absolute Wachstum der Stoffströme bislang entscheidend bremsen.

Inputorientierte Umweltpolitik zielt auf die auf die absolute Reduktion der Stoffströme auf der Inputseite, das heißt auf die Reduktion des gesamten Ressourcen-, Material- und Flächenverbrauchs ab (2). Im Vordergrund steht dabei das Bestreben, gesellschaftliche Suchprozesse in Richtung einer Erhöhung der gesamtwirtschaftlichen Ressourcenproduktivität zu lenken. $\mathrm{Zu}$ den Inputs zählen dabei alle Ressourcen, die während des gesamten Lebenszyklus eines Produktes benötigt werden, wie abiotische (zum Beispiel mineralische Rohstoffe, fossile Energieträger) und biotische Materialien (Biomasse), Böden und Flächen (verbraucht durch Erosion, Versiegelung, Zerschneidung), Kuppelentnahmen und verlagerte Massen (etwa nicht verwertete Rohförderung, Bodenaushübe), Wasser und Luft.

\section{Vorteile einer Inputorientierung}

Die aktuelle wissenschaftliche Diskussion zur Inputorientierung, im Englischen: Industrial Ecology, währt nun knapp eine Dekade und hat ihre Ursprünge Ende in der Debatte um den Industriellen Metabolismus Ender der 60er/70er Jahre. Die ökologischen und ökonomischen Vorteile einer solchen Strategie werden dabei unter anderem wie folgt begründet (3):

- Eine inputorientierte Umweltpolitik setzt statt an den Symptomen an den Ursachen der ökologischen Probleme an - an den Inputs und damit am Ressourcenverbrauch. Jeder menschliche Eingriff in Ökosysteme, jeder Abbau natürlicher Ressourcen führt zu unter Umständen irrever- siblen Veränderungen in der Natur. Jede Reduktion von Inputs reduziert Umwelteingriffe und damit mögliche externe Effekte.

- Eine inputorientierte Umweltpolitik ist effektiver als die konventionelle Umweltpolitik. Während die Anzahl der Stoffe auf der Inputseite bekannt und dadurch relativ einfach zu kontrollieren sind, kommt es auf der Outputseite zu räumlichen Schadstoffzerstreuungen, was die Kontrolle zum Teil unmöglich macht.

- Eine Orientierung am Ressourceninput bietet Möglichkeiten, umweltpolitische Aktivitäten zu bündeln, Umweltpolitik zu rationalisieren und zu verstetigen.

\section{Kontroverse Diskussion in Politik und Wissenschaft}

Diese Ziele werden in Wissenschaft und Politik allerdings sehr kontrovers diskutiert. Einerseits artikulieren sich Forderungen nach Senkung der Stoffströme auf internationaler und nationaler Ebene (4). So visiert die Nachhaltigkeitsstrategie der deutschen Bundesregierung etwa eine Steigerung der Energie- und Rohstoffproduktivität um den Faktor 2 bis 2020 an. Gleichzeitig wird von ökonomischer Seite eine Zielverfolgung „um jeden Preis“ und eine damit verbundene „Entmachtung der Ökonomie“ ,ja sogar eine ökologische „Zuteilungsgesellschaft“ befürchtet. Zu beachten ist allerdings, dass sich diese, teilweise fundamentale Kritik in der Regel an einzelnen Positionen entzündet, die irrtümlicherweise auf die gesamte Idee der Inputorientierung übertragen werden. Um aber das eigentliche innovative Potenzial einer Inputorientierung zu erkennen, ist es von entscheidender Bedeutung, sich mit den von naturwissenschaftlicher Seite formulierten Forderungen nach einer Inputorientierung gerade auch aus wirtschaftspolitischer Perspektive zu befassen.

\section{Stabilitäts- und verteilungs- politische Neutralität}

So gilt es zu fragen, inwieweit negative Effekte auf stabilitätspolitische Zielsetzungen vermieden werden können, insbesondere negative Beschäftigungs-, Wachstums- und Preisniveaueffekte. Grundlegend lassen sich zwei Positionen unterscheiden. Den Vertretern der Kostenschockthese zufolge werden die negativen Auswirkungen zusätzlicher Kostenbelastungen überwiegen. Die Anhänger der Porterschen Innovationsthese verweisen dagegen auf die positiven Effekte des Innovationsdrucks. Durch einen weitreichenden 
ökologischen Strukturwandel erhoffen sich die Befürworter der Innovationsthese insgesamt positive Beschäftigungseffekte sowie vernachlässigbare Auswirkungen auf den Preismechanismus. Die positiven Beschäftigungseffekte kommen bei Überwiegen der Effekte der Substitution von Ressourceninput durch Arbeit und die positiven Wachstumseffekte durch Lern- und Innovationseffekte zu Stande (vg. die Argumentation der neuen Wachstumstheorie). Allerdings sind für das Gelingen eines solchen Strukturwandels eine konsequente Wettbewerbspolitik, flexibel-dynamische Märkte, der Abbau von Regulierungen und die Öffnung aller Märkte notwendig, um Anreize für die Vielzahl erforderlicher Innovationen zu ermöglichen und Friktionen, die einem reibungslosen Strukturwandel im Wege stehen, weitestgehend abzubauen.

Ähnliches gilt für die verteilungspolitischen Effekte. Die vermutlich regressiven Effekte allgemeiner Ressourcenpreiserhöhungen können nur in Kauf genommen werden, wenn im Gegenzug die wirtschaftliche Dynamik zu positiven Beschäftigungsund Wachstumseffekten führt, die auch unteren Einkommensschichten zugute kommen. Die Vermutung der Regressivität gilt jedoch nicht für alle Ressourceninputkategorien. So kann bei einer Preiserhöhung bei der Flächennutzung eine progressive Belastung vermutet werden. Bei einem Instrumenteneinsatz mit erheblichen Verteilungseffekten müsste über eine Kompensierung dieser Effekte nachgedacht werden, da sonst die Umsetzung der gesamten Strategie gefährdet wäre. Allerdings sind auch die diskutierten kompensierenden Maßnahmen nicht unproblematisch (5). Die theoretisch notwendigen, funktionsfähigen Märkte sind in der Praxis allerdings nur unzureichend vorhanden. Zudem müssen international negative Verteilungseffekte für ärmere Länder, von denen erhebliche politische Widerstände zu erwarten sind, berïcksichtigt werden.

\section{Resümee: Ordnungskonforme Strategie überhaupt möglich?}

Folgende Anforderungen an eine inputorientierte Strategie ergeben sich aus einer wirtschaftspolitischen Analyse:

- Inputorientierte Zielsetzungen sind nur - langfristig kalkulierbar und demokratisch abgesichert - als Poppersche Strategie der Stückwerkreform umzusetzen. Der konkrete Instrumenteneinsatz müsste revidierbar gestaltet werden, so dass auf etwaige makroökonomische Fehlentwicklungen reagiert werden könnte.
- Dabei sind Friktionen eines ökologischen Strukturwandels nur bei hinreichender Flexibilität und Offenheit aller Märkte zu bewältigen. Verfestigte Marktstrukturen stehen einer notwendigen, auf einer Vielzahl von Innovationen basierenden Dynamik entgegen.

- Mittelfristig am ehesten umsetzbar wäre es, mit dem größten Problembereich mit der höchsten Akzeptanz zu beginnen. Die Zielgrößen müssten jedoch sehr allgemein gehalten werden, um eine ordnungspolitisch nicht gewünschte Feinsteuerung der Wirtschaftsstruktur zu vermeiden. Selektive Branchen- oder Produktziele sollten zum Beispiel ausgeschlossen sein. Aus diesen Überlegungen heraus erscheint es am geeignetsten, mit Reduktionszielen beim Energieinput beziehungsweise beim Flächen- und Grundwasserverbrauch zu beginnen, da eine Reduktion dieser Inputs eine breite Wirkung auf die gesamten Stoffströme entfalten würde.

- Marktwirtschaftliche Instrumente sind vorzuziehen. Eine administrative Verteilung der Stoffnutzungsrechte ist zu vermeiden und den Märkten zu überlassen. Die Zielgrößen sind dabei langfristig festzulegen, so dass Erwartungen stabilisiert werden und Innovatoren eine feste Planungsgrundlage haben (Vorrang der Ordnungs- vor der Prozesspolitik).

- Eine alleinige Konzentration auf das Volumen der Stoffströme abstrahiert von lokalen Umweltproblemen und unterschiedlichen Toxizitäten. Insofern können die Ansätze einer Dematerialisierung immer nur im Zusammenhang mit anderen Konzepten diskutiert werden. Zielführend kann nur ein dualer Ansatz sein , der problemspezifische und qualitative mit volumenorientierten Zielsetzungen und Indikatoren verbindet (6).

\section{Umsetzbarkeit im politischen Prozess?}

Allerdings bedürfen inputorientierte Zielsetzungen

- der allgemeinen politischen Akzeptanz, die insbesondere durch die normativen Voraussetzungen (Akzeptanz des Vorsichtsprinzips) schwierig ist.

- erheblicher Informations- und Motivationsmaßnahmen, um kognitive, soziologische und psychologische Barrieren zu überwinden.

- der politischen Akzeptanz für eine weitreichende Flexibilisierung und Deregulierung der Märkte als Vorbedingung eines reibungslosen Strukturwandels.
Andererseits stellen Entscheidungen über inputorientierte Zielsetzungen ordnungspolitische Grundkonsensentscheidungen dar, die zumeist unter einem Rawlsschen „Schleier des Nichtwissens" getroffen werden müssen. Dies erhöht wiederum ihre Chance, allgemein akzeptiert zu werden. Existieren tatsächlich gesamtgesellschaftliche Präferenzen für diese Zielsetzungen, werden in Direktdemokratien größere Chancen für eine Einigung auf grundlegende Zielsetzungen vermutet, während in repräsentativ-parlamentarischen Demokratien die Gefahr einer Deformierung der Zielsetzungen durch verteilungspolitische Konflikte zu befürchten ist (7). Darüber hinaus müsste diskutiert werden, ob eine konkrete Umsetzung inputorientierter Strategien nicht in unabhängigen Institutionen erfolgen sollte.

\section{Anmerkungen}

(1) Vgl. Bringezu, S./ H. Schütz: Total Material Requirement of the EU. Technical Report 55, Kopenhagen, 2001 (2) Vgl. grundlegend hierzu Stewen, M.: Inputorientierte Umweltpolitik in der Sozialen Marktwirtschaft - Wirtschaftspolitische Analyse einer "nachhaltigen" Reduktion der Stoffströme, Baden-Baden, 2003.

(3) Vgl. Hinterberger, F./ F. Luks/ M. Stewen: Ökologische Wirtschaftspolitik. Zwischen Ökodiktatur und Umweltkatastrophe. Berlin, 1996, $71 \mathrm{ff}$.

(4) Vgl. z.B. die Forderungen nach einem Faktor 10, z.B. bei Schmidt-Bleek: Wieviel Umwelt braucht der Mensch? Berlin u.a., 1994.

(5) Vgl. zu den Verteilungseffekten inputorientierter Umweltpolitik Luks, F./ M. Stewen: Why Biophysical Assessments Will Bring Distribution Issues to the Top of the Agenda, in: Ecological Economics, Vol. 29, 1999, $33 \mathrm{ff}$.

(6) Vgl. Bleischwitz, R: Ressourcenproduktivität - Innovationen für Umwelt und Beschäftigung. Berlin u.a., 1998, 3lff.

(7) Vgl. Stewen, M.: Grundkonsens- und Einzelfallentscheidungen in der Umweltpolitik - Ein Beitrag zur Neuen Politischen Ökonomie umweltpolitischer Entscheidungsprozesse. Zeitschrift für Umweltpolitik und Umweltrecht, 23/3, 2000, 409-439.

\section{Der Autor}

Dr. Marcus Stewen ist Projektmanager mit Schwerpunkt Umwelt- und Ressourcenschutz bei der Kreditanstalt für Wiederaufbau (KfW) und Mitglied im Sustainable Research Institute Wien (SERI).

Kontakt: E-Mail: marcus.stewen@seri.de 
(c) 20I0 Authors; licensee IÖW and oekom verlag. This is an article distributed under the terms of the Creative Commons Attribution Non-Commercial No Derivates License (http://creativecommons.org/licenses/by-nc-nd/3.o/), which permits unrestricted use, distribution, and reproduction in any medium, provided the original work is properly cited. 